\title{
Unsymmetrical shear loading and its influence on the frictional shakedown of incomplete contacts
}

\author{
D Dini and D A Hills* \\ Department of Engineering Science, University of Oxford, UK
}

\begin{abstract}
Although incomplete contacts can never shake down to a completely adhered state, the self-development of residual frictional shearing tractions can reduce significantly the size of the slip zone. This phenomenon is demonstrated using the Hertz-Cattaneo contact.
\end{abstract}

Keywords: unsymmetrical shear loading, frictional shakedown, incomplete contacts

\section{INTRODUCTION}

This paper is concerned with static incomplete contacts, subjected to a cyclically varying shear force. It is therefore of direct relevance to the study of fretting fatigue in a wide range of problems. For example, the contact between the dovetail root of a gas turbine fan blade and disc falls into this class and is currently giving rise to difficulties in many designs. A thorough understanding of the properties of this class of contact, and, in particular, the stick-slip zone regime, traction distribution and relative slip displacement between the contacting bodies, all of which are of primary significance in predicting fretting damage, is of great importance. Incomplete contacts, i.e. those where the contacting bodies are smooth and convex, so that the contact pressure always falls continuously to zero at the contact boundary, will always suffer partial slip when a shearing force, less than that needed to cause sliding, is sequentially applied. In this paper the most celebrated solution is considered for this class of problem, that for the Hertzian contact, first discovered by Cattaneo in 1938 [1] and rediscovered by Mindlin some 10 years later [2]. The intention is to analyse the nature of the slip displacement developed throughout the loading cycle and to show that, even though there is slip in the steady state, the change in slip displacement in part of the initially slipping region may be infinitesimal, so that no damage will occur there.

Cattaneo analysed the contact only under a monotonically increasing shearing force. Mindlin went on to complete a more exhaustive treatment of the problem

The MS was received on 25 November 2003 and was accepted after revision for publication on 9 February 2004.

* Corresponding author: Department of Engineering Science, University of Oxford, Parks Road, Oxford OX 1 3PJ, UK. but concentrated on the three-dimensional Hertzian contact, where the effects of unloading and reloading were treated $[3,4]$. This is perhaps slightly surprising because the superposition technique employed is inexact in these cases [5]. One element of the problem which, although well understood, does not seem to have been pursued in the intervening years is the question of the self-development of residual shearing tractions, or frictional shakedown, in cases when the shear loading cycle is unsymmetrical.

Before turning to a detailed analysis of the problem, the general concept of frictional shakedown is now introduced. Shakedown in continuum plasticity problems is well known. It occurs under cyclic loading and is the self-generation of a protective residual stress state which tends always to alleviate the severity of the applied state of stress, as measured by a yield criterion, during subsequent cycles. The process is quite distinct from work hardening, the elevation of the material's yield stress, which may proceed separately, and both tend to reduce the tendency of the component to become plastic. An analogue may be drawn between plastic flow and slip present at an interface between contacting components; at one level, yielding may be regarded as the manifestation of internal slip within the continuum, rather than macroscopic slip at an interface. At a more practical level, parallels may be drawn between the effects of plastic shakedown and work hardening, and what happens at a contact interface under cyclic loading, particularly with a varying applied shearing force. For example, if the analogy set out above is carried through, the surface modification which occurs under cyclic loading, and which leads to an increase in the coefficient of friction, is similar to work hardening. Further, if, during the first cycle of loading where the contact is subject to a monotonically increasing shearing force, the contact interface enters a state of partial slip, 
but there is no corresponding reverse slip during unloading, upon removal of the load a residual interfacial shearing traction will remain. Clearly, this is very similar to what happens during plastic shakedown and means that, upon reloading the contact, the residual shearing tractions present will alleviate the tendency to slip. Thus, there is the possibility that, after a finite number of cycles, what started as a contact in partial slip will eventually become fully stuck. If further terminology is borrowed from plasticity theory, the highest load that may be supported by the contact without there ever being any slip may be thought of as the elastic limit, while the highest load that may be supported without there being any slip in the steady state may be considered to be the shakedown limit.

Now the two fundamental classes of contact, namely complete and incomplete, are looked at. In a complete contact, where the size of the contact patch is independent of the applied load, the contact pressure is extremely large at the boundary (indeed, an elastic asymptotic analysis shows it to become infinite), so that a residual interfacial shearing traction, for a finite normal contact load, may be supported everywhere along the interface. A recent example of such a contact has been studied [6], and both the 'elastic limit' and the 'shakedown limit', as defined above, have been found. Now, in an incomplete contact, as stated at the outset, the contact pressure must fall continuously to zero at the boundaries of the contact. It follows that no residual shearing traction may be sustained there and that therefore, formally, the 'elastic limit' and 'shakedown limit' of this class of problem are both always identically zero. However, it is worth returning to the question of why the presence of 'slip' is so interesting. It is known that fretting, the minute interfacial slip that occurs in some contacts, has a very deleterious effect on the nucleation of fatigue cracks (hence 'fretting fatigue'). However, the surface damage occurs precisely because there is finite slip between the bodies, giving rise to surface modification. It is not the intention in the present paper to speculate on the nature of that modification, but it is clear that, without relative slip, modification cannot occur. What will be shown in this paper is that, in a range of circumstances, incomplete contacts exist in a steady state where, although in one part of the contact patch they show incipient slip, the actual slip displacement arising there is vanishingly small. For practical purposes, they have shaken down to a state where stick is maintained over a larger fraction of the contact.

Finally, before proceeding to the example problem, one further observation is made. This is that, in addition to the 'frictional shakedown' phenomenon being addressed here, contacts may exhibit classical 'plastic shakedown'. In this article, plastic shakedown is not considered at all. However, it is important to recognize that the presence of residual interfacial tractions does change the nature of the local internal state of stress within the contacting bodies. Thus, when the strength of the contact, as determined by setting the most severe state of stress to the yield condition is found, its value will be affected by frictional shakedown. In real contacts, plastic and frictional shakedown processes may proceed in parallel.

\section{FORMULATION}

Figure 1 shows schematically a Hertzian contact, the loading to which it is subjected and the resulting interfacial shearing traction at turning points in the cycle. A fully reversing shearing force $Q(t)$ is applied, and the resulting shear traction distribution $q(x)$ present at the turning points of the loading waveform, i.e. at $\pm Q^{\max }$. In the figure, $f$ is the coefficient of friction, $a$ is the contact half-width, $P$ is the normal contact load, and $p(x)$ and $q(x)$ are the direct and shearing traction distributions respectively. A detailed explanation of the Cattaneo-Mindlin calculation may be found in the original papers, or in many postgraduate textbooks. For example Johnson's book [7] has a good description of the problem, and explanations have also been given by Hills and Nowell [8] or Waterhouse [9]. It is clear that, in this case, there can be no residual shearing tractions, because any residual shear which ameliorated the tendency to slip in one half-cycle would exacerbate it in the other, so that in each half-cycle of loading the shearing traction is simply reflected. Furthermore, the steady state solution is the same as the solution in the first quarter-cycle of loading. (This is entirely analagous to the problem of, for example, a beam in fully reversed bending, where clearly no residual stress can arise and therefore no plastic shakedown can take place.) When the shear loading is unsymmetrical, however, the problem changes qualitatively, and this is the problem that will be studied here.

The force history of the problem to be analysed, where the applied shearing force oscillates between $Q^{\max }$ and $Q^{\min }$, with $\Delta Q=Q^{\max }-Q^{\min }$, is shown schematically in Fig. 2. Without loss of generality, it is required that

$$
-Q^{\max }<Q^{\min } \leqslant Q(t) \leqslant Q^{\max }>0
$$

and the traction distribution may be tracked out as a function of time using the technique first developed by Cattaneo. The principal point to note is that, at each turning point in the $Q(t)$ curve, the increment of slip displacement has an opposite sign to the shear traction, so that stick ensues everywhere, thereby freezing in the shear tractions present at that point. In order to determine the influence of a finite reduction in the absolute magnitude of the shear (e.g. point C in Fig. 2), an additional corrective term must be applied to the new 

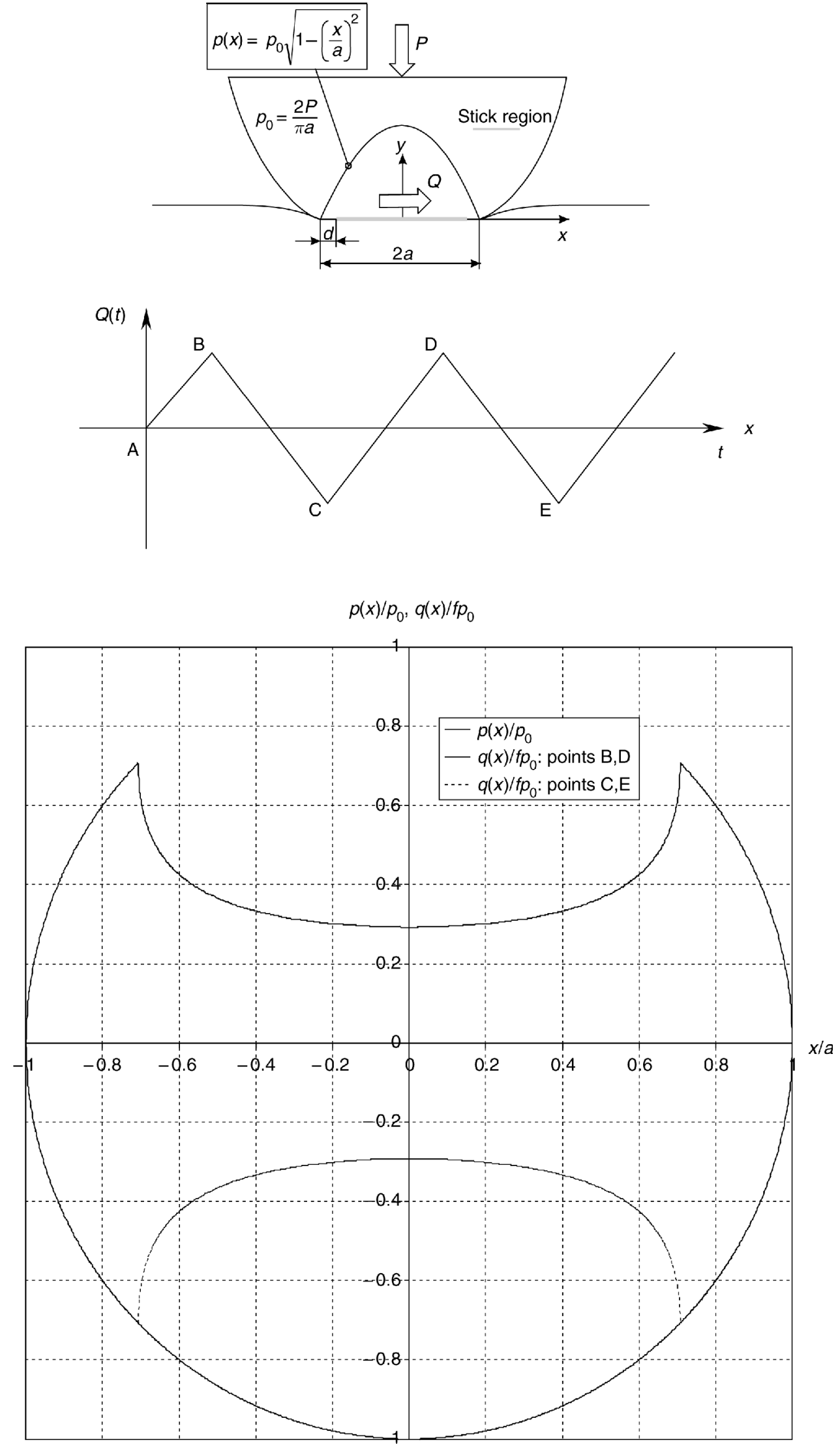

Fig. 1 Contact pressure distribution and shearing traction distribution present on a Hertzian contact surface subject to fully reversing loading $Q^{\max } /(f P)= \pm 0.5$ 


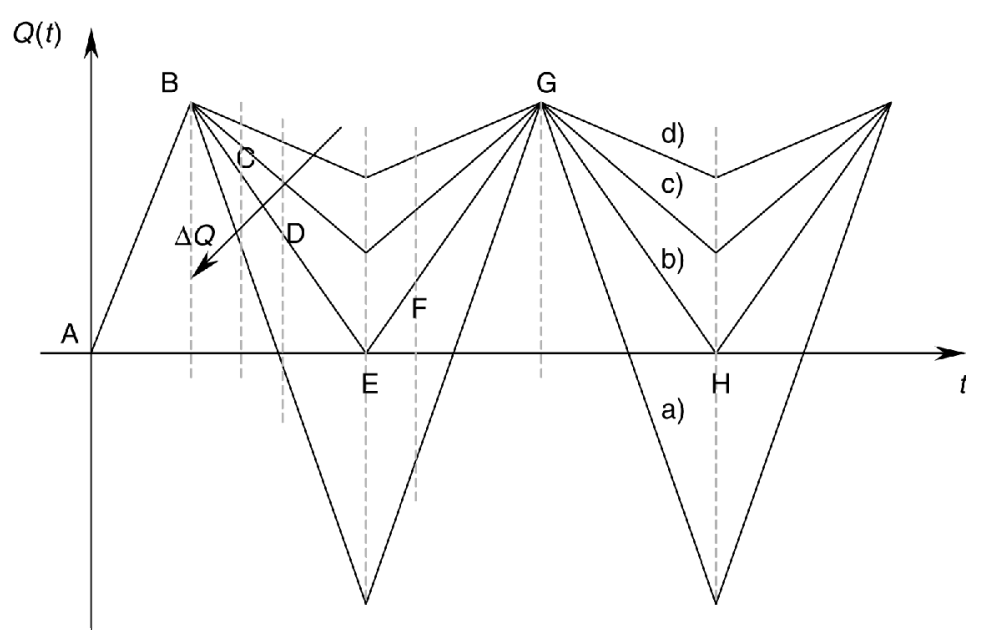

Fig. 2 Example loading histories: curve (a), $Q^{\min }=-Q^{\max }$; curve (b), 0 ; curve (c), $Q^{\max } / 2$; curve (d), $3 Q^{\max } / 4$

stick zone while considering reverse slip at the edges of the contact. This technique has already been exploited by many analysts, and a detailed description has been given by, for example, Hills et al. [10]. The same procedure can be applied at each turning point in the loading cycle so that, upon reloading, a subsequent finite increment in the shear force (point F in Fig. 2) will cause a further change in the sign of displacements within the slip region and, again, the shear traction distributions may be obtained by introducing a further corrective term to the solution derived for $Q=Q^{\text {min }}$.

\section{RESULTS}

Suppose that two cylinders are first pressed together, and the normal contact force held constant. A monotonically increasing shearing force is applied, up to $Q^{\max }$, giving rise to a receding stick zone, and a slip zone whose maximum extent is $d_{0}$, given by $d_{0} / a=1-$ $\sqrt{1-Q^{\max /(f P)}}$ [1]. The shearing force is now decreased infinitesimally, so that stick ensues everywhere, and a finite reduction in shearing force to $Q(t)$ results in a regime of backslip of extent $d^{\text {unload }}(t)$, given by

$$
\frac{d^{\text {unload }}(t)}{a}=1-\sqrt{1-\frac{\Delta Q}{2 f P}}
$$

where

$$
\Delta Q=Q^{\max }-Q(t)
$$

The shearing force is reduced until the lower limit $Q^{\min }\left(>-Q^{\max }\right)$ is reached. The extent of the region of backslip is $d_{1}$, with $d_{1} / a=1-\sqrt{1-\Delta Q /(2 f P)}$, and intermediate distributions of shearing traction are shown in Fig. 3.
Suppose, now, that the shearing force is increased infinitesimally. This will result instantaneously in adhesion everywhere, followed by a new region of forward slip developing; for $Q^{\min }<Q(t)<Q^{\max }$, its extent, $d^{\text {reload }}(t)$, is given by

$$
\frac{d^{\text {reload }}(t)}{a}=1-\sqrt{1-\frac{Q(t)-Q^{\text {min }}}{2 f P}}
$$

The evolution of the shear traction distributions during the reloading process is plotted out for representative values of $Q(t)$ in Fig. 4. Note, particularly, that the extent of forward slip, if $Q(t)=Q^{\max }-\varepsilon$, is $d_{1}$; i.e. it approaches the extent of maximum reverse slip present at the minimum load. Note, too, that in the region $d_{1} \leqslant x \leqslant d_{0}$, the shearing traction is everywhere infinitesimally below its value during the first quarter-cycle, when the shearing force was initially increased monotonically to $Q^{\max }$. It follows that, if $\varepsilon \rightarrow 0$, although formally the slip zone will now extend to its original size, the increase in slip displacement during this change will itself be infinitesimal. Thus, for practical purposes, whether the shearing force is increased identically with $Q^{\max }$ or stopped just short, the change in slip displacement in the range $d_{1} \leqslant x \leqslant d_{0}$ is either zero or negligible. It follows that some frictional shakedown has occurred. This is an important feature of this analysis and one which appears not to have been observed before. It is clear that, for practical purposes, frictional shakedown to a state of extended adhesion can occur in incomplete contacts.

Further practical implications of this result are now considered. First, the maximum extent of the steady state slip zone (whether during the forward or reverse slipping parts of the cycle) is $d_{1}$, and this is governed by $\Delta Q$ alone. Further, since it is the range of stresses (however quantified) which is thought to do the damage 


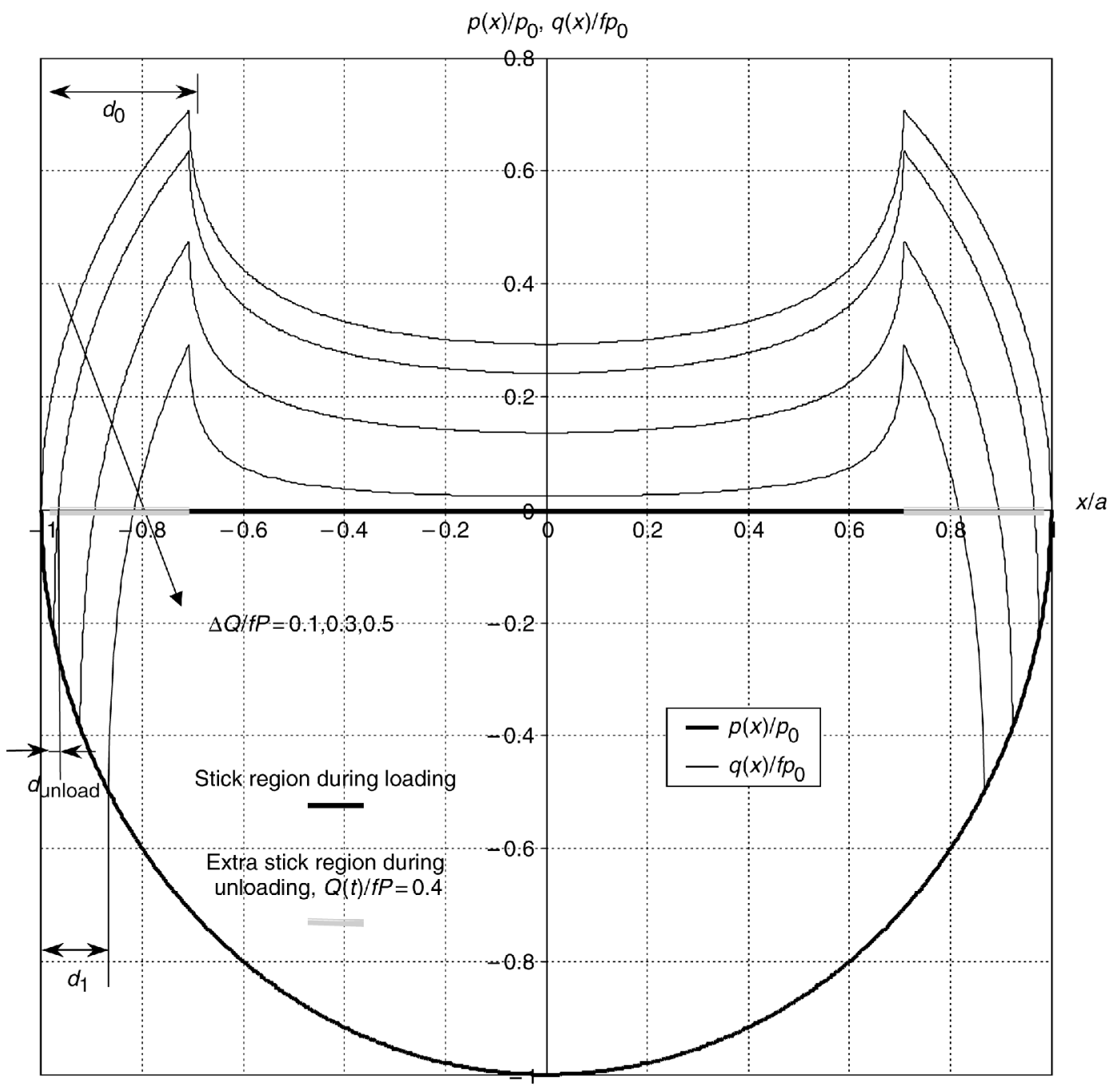

Fig. 3 Example shearing traction distribution during the unloading phase, $Q^{\max } /(f p)=0.5$, and unloading to $Q^{\min } /(f p)=0.4,0.2$ and 0.0

during crack nucleation, it is noted that, at the extremes of the shearing force, the traction components of stress are given by

$$
\begin{aligned}
& \frac{p(x)}{p_{0}}= \pm \frac{q(x)}{f p_{0}}=\sqrt{1-\left(\frac{x}{a}\right)^{2}}, \\
& 0 \leqslant x \leqslant d_{1}, d_{1}<d_{0} \text { if } Q_{\min }<-Q_{\max }
\end{aligned}
$$

Further, the component of stress parallel to the surface due to the normal contact pressure alone $\sigma_{x x}^{P}(s)$ is identical with the contact pressure, from Way's theorem, leaving only the contribution from the shearing traction, $\sigma_{x x}^{Q}(s)$, to be found. This is profoundly dependent on the effect of the locked-in shearing traction induced, and hence on $Q^{\max }$.

It is difficult to make further progress on the application of this result to the strength of a fretting contact without making an assumption about the qualities of the state of stress which affect crack nucleation. This will be achieved, therefore, in two ways. Firstly, the strength of the contact will be found as determined by first yield. The von Mises contours have been plotted for the state of stress, and these were used to determine the maximum load that can be sustained before the onset of yield. The strength or elastic limit of the contact, $p_{0} / k$, where $k$ is the yield stress in pure shear and $p_{0}$ the peak Hertzian contact pressure (see Fig. 1), was found as a function of the $f$ and $Q^{\max } /(f P)$ (see solid curves in Fig. 5). This result is therefore appropriate for both a monotonically increasing shearing force and a fully reversing shearing force, where no frictional shakedown can occur. For small values of $Q / P$ the most severe state of stress occurs on the surface, and it is only weakly dependent on $Q / P$, giving rise to an elastic limit $p_{0} / k \approx 3.1$. Secondly, further results have been 


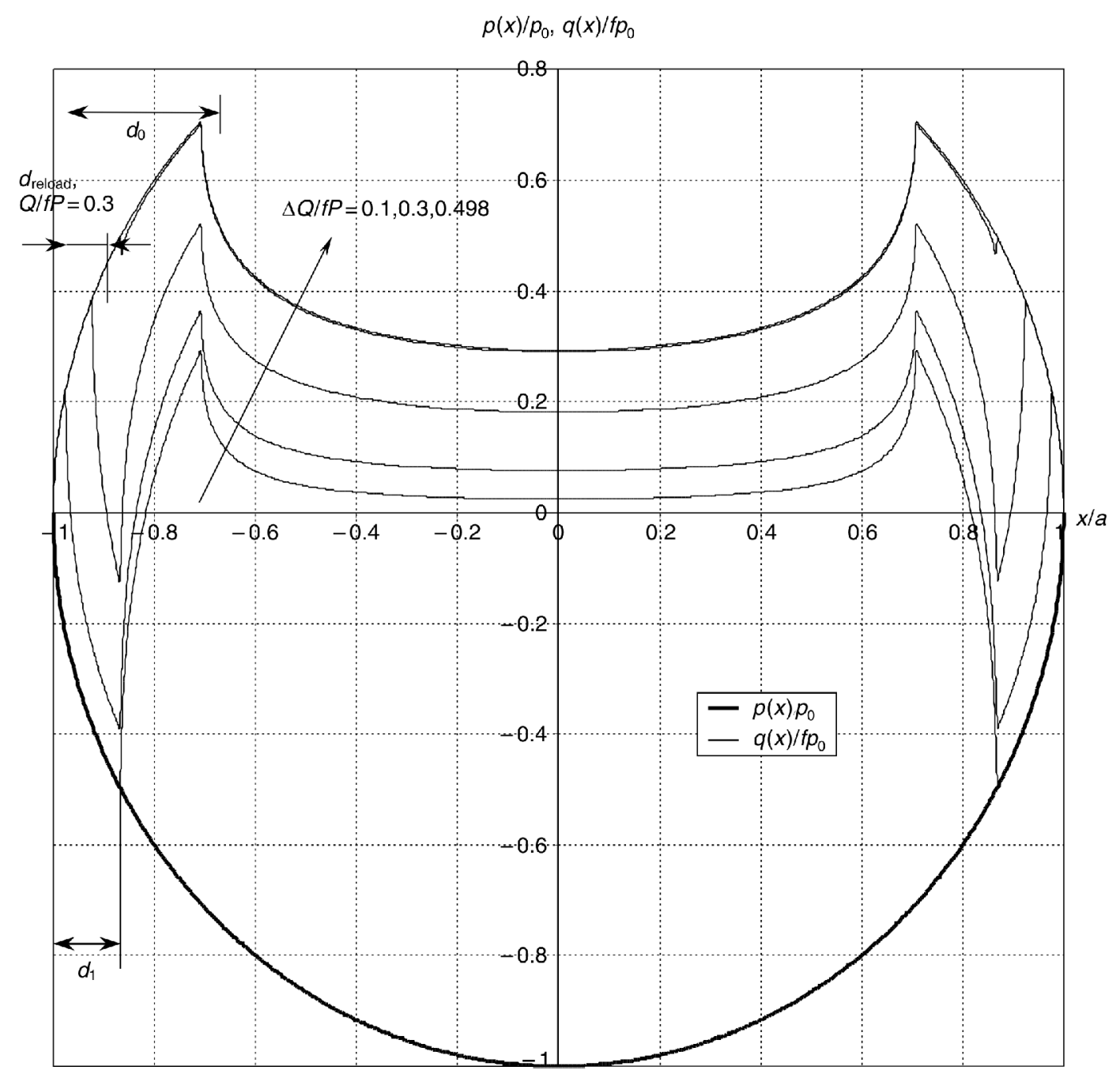

Fig. 4 Example shear traction distribution during reloading $Q^{\max } /(f p)=0.5, Q^{\min } /(f p)=0$ and now $Q(t) /(f p)=0.1,0.3$ and 0.498

produced for the case where the contact is suffering an unequal shearing force, and hence frictional shakedown ensues. It is not possible to produce fully comprehensive plots, as there are now three independent variables, namely $f, Q^{\max } /(f P)$ and $Q^{\min } /(f P)$. Nevertheless, the comparisons carried out show that, when the strength is surface limited and attention is restricted to the slip region, the elastic limit of the contact is higher when the slip region is smaller (because of shakedown). This is shown by the dashed lines in Fig. 5 which represent the contact strength $p_{0} / k$ in the steady state slip region for a coefficient of friction, $f=0.6$.

Although the effect in this instance of frictional shakedown on the elastic limit is small, other effects are not. In particular, the damage zone is localized and the slip displacements at the edge of the contact are smaller.

\section{CONCLUSION}

The effects of frictional shakedown on the strength of the Hertz-Cattaneo contact have been found. Note that the same ideas will carry over to other incomplete contacts such as the flat and rounded punch; in particular, the size of the steady state slip region is dependent on the range of shear stresses only, and not on its maximum value. This means:

(a) that the region of slip in the steady state will, except in the case of fully reversing loading, be smaller than the initial value,

(b) that the slip displacement will be smaller and

(c) that the contact strength will slightly improve. 


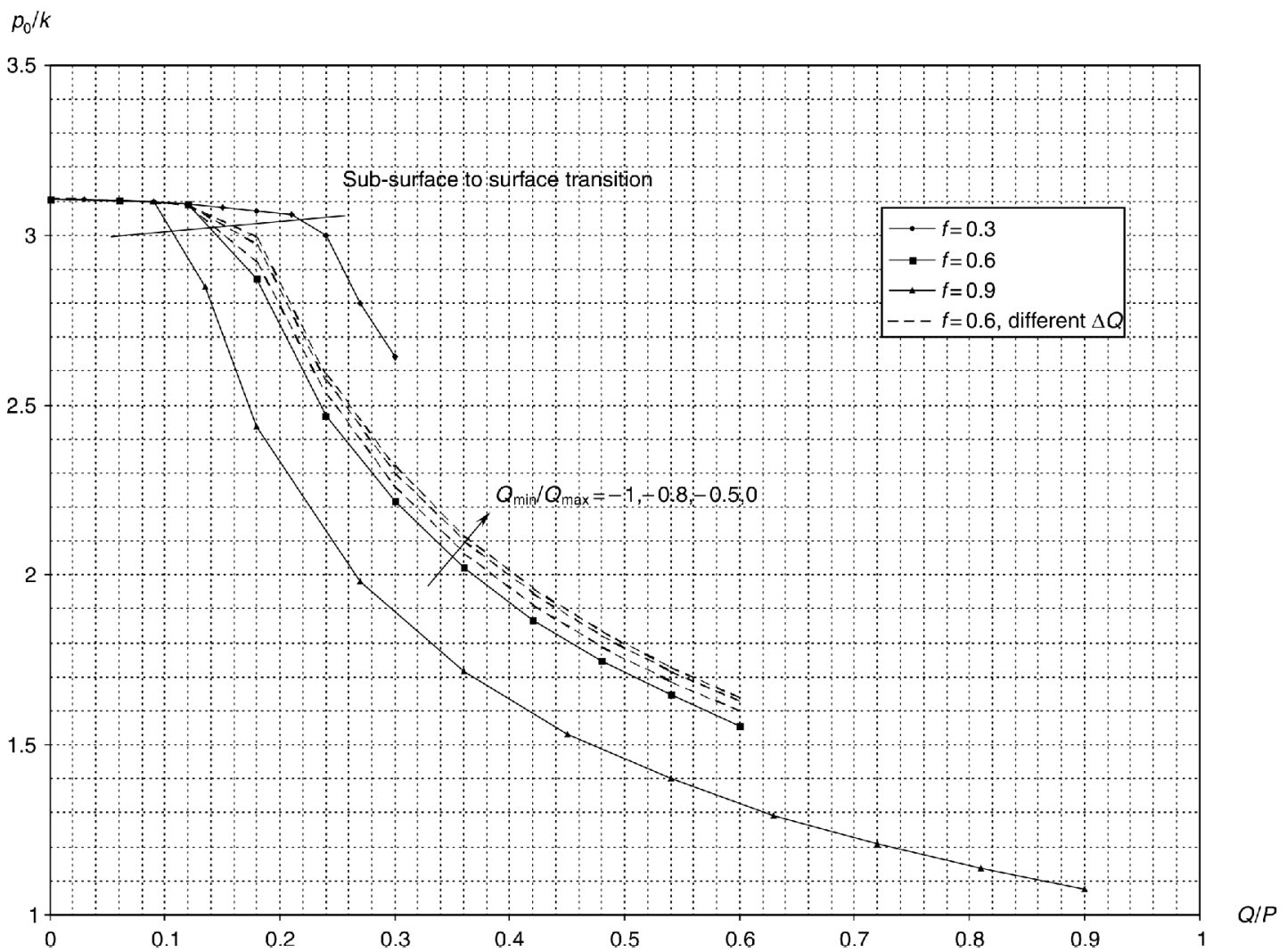

Fig. 5 The elastic limit for a partial slip contact: - , strength for fully reversing loading; - - , $f=0.6$ and $Q^{\min } / Q^{\max }=-0.8,-0.5$ and 0

The first two points show that, in the steady state, the region of steady state slip is very localized; secondly, the magnitude of the slip displacements is much reduced.

\section{ACKNOWLEDGEMENT}

David A. Hills would like to thank Dr Ian Archer and Dr Brian Gasser (co-proctor and clerk to proctors respectively) for indulging him in studying mechanics when he should have been upholding the University Statutes.

\section{REFERENCES}

1 Cattaneo, C. Sul contatto di due corpi elastici: distribuzione locale degli sforzi. Rc. Accad. Naz. Lincei, 1938, 27, 342-348, 434-436, 474-478.

2 Mindlin, R. D. Compliance of elastic bodies in contact. $J$. Appl. Mechanics, 1949, 16, 259-268.
3 Mindlin, R. D., Mason, W. P., Osner, T. F. and Deresiewicz, $\mathbf{H}$. Effects of an oscillatory tangential force on the contact surfaces of elastic spheres. In Proceedings of the 1st National Congress of Applied Mechanics, 1951, pp. 203-208.

4 Mindlin, R. D. and Deresiewicz, H. Elastic spheres in contact under varying oblique forces. J. Appl. Mechanics, 1953, 75, 327-344.

5 Munisamy, R. L., Hills, D. A. and Nowell, D. Static axisimmetrical Hertzian contacts subject to shearing forces. J. Appl. Mechanics, 1994, 61, 278-283.

6 Saez, R., Mugadu, A., Fuenmayor, F. J. and Hills, D. A. Frictional shakedown in a complete contact. J. Strain Analysis, 2003, 38 (4), 329-338.

7 Johnson, K. L. Contact Mechanics, 1985 (Cambridge University Press, Cambridge).

8 Hills, D. A. and Nowell, D. Mechanics of Fretting Fatigue, 1994 (Kluwer, Dordrecht).

9 Waterhouse, R. B. Fretting Fatigue (Ed. R. B. Waterhouse), 1981 (Applied Science, Barking, Essex).

10 Hills, D. A., Nowell, D. and Sackfield, A. Mechanics of Elastic Contacts, 1993 (Butterworth-Heinemann, Oxford). 